\title{
Epigenetic Dysregulation in Laryngeal Squamous Cell Carcinoma
}

\author{
Thian-Sze Wong, Wei Gao, Zeng-Hong Li, Jimmy Yu-Wai Chan, and Wai-Kuen Ho \\ Department of Surgery, Queen Mary Hospital, The University of Hong Kong, 102 Pokfulam Road, Hong Kong \\ Correspondence should be addressed to Thian-Sze Wong, thiansze@gmail.com
}

Received 9 December 2011; Revised 16 February 2012; Accepted 16 February 2012

Academic Editor: Michiel W. M. van den Brekel

Copyright ( 12012 Thian-Sze Wong et al. This is an open access article distributed under the Creative Commons Attribution License, which permits unrestricted use, distribution, and reproduction in any medium, provided the original work is properly cited.

\begin{abstract}
Laryngeal carcinoma is a common head and neck cancer with poor prognosis. Patients with laryngeal carcinoma usually present late leading to the reduced treatment efficacy and high rate of recurrence. Despite the advance in the use of molecular markers for monitoring human cancers in the past decades, there are still no reliable markers for use to screen laryngeal carcinoma and follow the patients after treatment. Epigenetics emerged as an important field in understanding the biology of the human malignancies. Epigenetic alterations refer to the dysregulation of gene, which do not involve the alterations of the DNA sequence. Major epigenetic changes including methylation imbalance, histone modification, and small RNA dysregulation could play a role in the development of human malignancies. Global epigenetic change is now regarded as a molecular signature of cancer. The characteristics and behavior of a cancer could be predicted based on the specific epigenetic pattern. We here provide a review on the understanding of epigenetic dysregulation in laryngeal carcinoma. Further knowledge on the initiation and progression of laryngeal carcinoma at epigenetic level could promote the translation of the knowledge to clinical use.
\end{abstract}

\section{Introduction}

Head and neck squamous carcinoma is estimated to be the sixth most common malignant tumor worldwide. Of which, laryngeal carcinoma is the second most common head and neck squamous carcinoma [1]. According to the global cancer statistics in 2008 , the age-standardized incidence rate (ranged from age $0-74$ ) is 5.5 per 100,000 in men and 0.6 per 100,000 in women in developed areas; in less developed area, the incidence rate is 3.5 per 100,000 in men and 0.6 per 100,000 in women [2]. It is estimated that there are 12,740 new patients (male 10,160 and female 2,580, resp.) suffering from laryngeal carcinoma in the United States in 2011 (accounting for $0.7 \%$ of the total new cancer cases). It also accounts for about $0.6 \%$ estimated cancer-related deaths in the United States [3]. In Hong Kong, laryngeal carcinoma took the third place in the incidence of the head and neck cancer in 2009 [4].

Most of the laryngeal carcinoma patients are male [5]. Moreover, the majority of the patients lie in the middleaged group [6]. The definite cause of laryngeal carcinoma is not yet determined, while some risk factors are believed to be linked with the development of the disease. Tobacco and alcohol consumption are the primary aetiologic factors [6$8]$. Chronic laryngeal inflammation induced by irritants and prolonged voice abuse could also contribute to the development of laryngeal carcinoma [9]. Recently, it was demonstrated that viral infection is a plausible cause for laryngeal carcinoma. However, the causal link between HPV and laryngeal carcinoma remains controversial. Human papilloma virus (HPV) types $6,11,16,18$, and 33 are detected in patients with laryngeal carcinoma [10]. Among all the detected HPV subtypes, the infection prevalence of HPV type 16 is much higher than the infection of the other types [11]. The link between HPV infection and laryngeal carcinoma development is affected by geographical factor and was confirmed in two large case-control studies [12].

Diagnosis of laryngeal carcinoma depends on the following: symptoms (e.g., hoarseness, voice changes, and feeling of a lump in the throat); signs (e.g., neck mass and laryngeal tenderness); assistant examination (including CT scan, electronic laryngoscopic detection, and pathological biopsy) [13]. Among these, the result of pathological biopsy, which can supply a histological evidence, is the golden standard for the diagnosis of laryngeal carcinoma. Histologically, most of 
the laryngeal carcinomas are squamous cell carcinoma. Morphological changes including dyskeratosis, laryngeal intraepithelial neoplasia, atypia, and dysplasia are lesions with high chance to transform from mild dysplasia to carcinoma in situ [14]. In comparison, minor squamous epithelial changes such as squamous metaplasia, squamous hyperplasia, pseudoepitheliomatous hyperplasia, keratosis, and parakeratosis may be found in the laryngeal region without subsequent malignant transformation [14].

In order to find out the definite cause of laryngeal carcinoma and the available index for the diagnosis of this disease, researchers put their attention on the research of molecular markers. Since 1983, the epigenetics of human cancer draw the researchers' attention [15]. It is now recognized that epigenetic markers including hypermethylated DNA and oncogenic microRNA could be used as screening markers for human cancers. Further, epigenetic drugs including decitabine, zebularine, and TSA are under examination for use as anticancer drug by reversing the altered epigenetic traits of cancer cells. In comparison with the epigenetic studies in other solid cancers, studies on laryngeal carcinoma are comparatively fewer. In this paper, we summarized the current knowledge on epigenetic alterations in laryngeal carcinoma from the published reports.

\section{Epigenetic Alterations in Human Malignancies}

$\mathrm{Wu}$ and Morris defined epigenetics changes as "changes in gene function that are mitotically and/or meiotically heritable and that do not entail a change in DNA sequence" [16]. Thus, an epigenetic trait is developed from the epigenetic changes in gene expression without the alteration of DNA sequence [17]. In general, epigenetic alterations broadly cover all the changes resulting in gene expression regulation without interfering with the DNA sequence at genetic levels [18]. In human cancers, common epigenetic alterations include DNA methylation, histone modification, and noncoding RNA dysregulation [14, 15]. In the past decade, studies on the abnormal epigenetic changes involved in human malignancies are numerous leading to the development of epigenetic markers and therapeutic targets at epigenetic level.

\subsection{Aberrant DNA Methylation Changes in Human Cancers.} In 1969, Griffith and Mahler proposed that modification of DNA base is a possible way to modulate gene expression [19]. In mammalian cells, modification of the cytosine residue is the predominant DNA modification, especially in the cytosine of CpG dinucleotide [20]. In human genome, the CpG dinucleotides are particularly rich in large repetitive sequence (e.g., centromeric repeats) and gene regulatory regions (e.g., $\mathrm{CpG}$ islands). Aberrant CpG island methylation is catalyzed by mammalian DNA methyltransferase and is usually found in the upstream region of the transcription start sites. CpG island methylation could block the accessibility of transcription factor to the promoter region of the tumor suppressor genes [21]. Additionally, the methylated sequence itself could recruit histone-modifying protein which induces gene silencing by changing the chromatin structure from active open chromatin structure to condensed closed chromatin structure [22, 23]. Approximately, $60 \%$ of the protein-coding genes in the human genome contain the $\mathrm{CpG}$ islands at their regulatory regions [24]. In cancer cells, $\mathrm{CpG}$ islands become methylated and there is de novo addition of methyl group to the C5 position of the cytosine ring [25]. DNA methylation is the most studied epigenetic change in head and neck cancers. In head and neck cancer, aberrant promoter methylation has been found in a wide variety of genes including p14ARF, p15, p15INK4B, p16, p16INK4A, ATM, DCC, DAPK, MINT1, MINT2, MINT27, MINT31, RARbeta, CDH1, cyclin A1, cytoglobin, RASSF1A, LHX6, MLH1, MGMT, and CDKN2A [26-35]. These genes are involved in cell cycle, apoptosis, angiogenesis, cell-cell adhesion, migration, invasion, and metastasis [26-35]. Most of the studies focusing on the head and neck cancers employed a collection of tissues collected from different head and neck regions including nasopharynx, oral cavity, larynx, and hypopharynx. It is difficult to specify a methylation pattern which belongs to the laryngeal carcinoma alone. Here, we performed a review of the methylated genes with clearer association with laryngeal carcinomas.

2.1.1. Chromodomain-Helicase-DNA Binding Protein 5 Methylation. Chromodomain-helicase-DNA-binding protein 5 (CHD5) is involved in modifying chromatin structure. CHD5 protein contains chromatin remodeling, helicase, and DNA-binding motifs. Reduced CHD5 expression has been reported in neuroblastoma [36]. Mutation of CHD5 is a rare event. Loss of CHD5 was linked to the chromosomal deletion on $1 \mathrm{p} 36$ and promoter hypermethylation in neuroblastoma $[36,37]$. Chromosomal abbreviation at $1 p 36$ has been reported in head and neck carcinoma [38]. Loss of heterozygosity and instability of $1 \mathrm{p} 36$ could be detected in primary oral and laryngeal carcinomas [39]. Although loss of CHD5 has been shown in laryngeal carcinoma, the association between 1 p36 aberrations and CHD5 loss in laryngeal carcinoma has not yet been confirmed [40]. In laryngeal carcinoma cell lines, CHD5 functions as growth and invasion inhibitor [40]. CHD5 could stabilize the key regulator of head and neck carcinoma, P53, through activating expression of p19arf [41]. Recently, it was shown that promoter region of CHD5 was methylated in laryngeal carcinoma cell lines [40]. The epigenetic changes of CHD5 expression are reversible by demethylating agent. Demethylation treatment of laryngeal carcinoma cell line with 5-Aza-dC could restore CHD5 expression [40].

2.1.2. E-Cadherin Methylation. E-cadherin (cadherin 1, type 1 , E-cadherin (epithelial)) is a $97-\mathrm{kDa}$ transmembrane glycoprotein. Functional E-cadherin protein contains five extracellular cadherin repeats, a transmembrane region and a cytoplasmic tail. E-cadherin functions as adhesion protein of epithelial cells and is involved in cell attachment and the cell polarity and tissue architecture [42]. Loss of E-cadherin expression enhances head and neck cancer cell migration and increases the risk of metastasis [43]. Further, loss 
of E-cadherin could promote proliferation of head and neck cancer cells through activating epidermal growth factor receptor (EGFR) pathways [44]. In laryngeal carcinoma, reduced expression of E-cadherin has also been reported [45, 46]. Loss of E-cadherin is particularly associated with supraglottic carcinoma of the larynx and is associated with the aggressiveness of the cancers [47]. Downregulation of E-cadherin of supraglottic larynx carcinoma is associated with histological differentiation and/or lymph nodes metastases [48]. Loss of E-cadherin is considered as a good indication of regional lymph node metastasis in laryngeal carcinoma patients [49-51]. In addition, the expression level of E-cadherin is a potential indicator to predict the effect of overall treatment time in patients with supraglottic carcinomas [52]. In head and neck cancers, loss of E-cadherin in parallel with promoter hypermethylation is frequently reported and is suggested to be linked with the aggressive behavior of the cancers $[47,53]$. In a case control study with 235 patients with laryngeal and hypopharyngeal cancers, E-cadherin was reported as one of the most commonly methylated gene in laryngeal and hypopharyngeal cancers; however, no significant predictive value was found [54]. Recently, Marsit et al. demonstrated that methylated E-cadherin was only associated with head and neck patients who are light smokers [55].

2.1.3. P16 Methylation. P16 or cyclin-dependent kinase inhibitor $2 \mathrm{~A}$ is a cell cycle regulator controlling G1 to G2 cell cycle arrest. P16 could bind to cyclin-dependent kinase 4 protein (Cdk4) resulting in loss of interaction between Cdk4 and cyclin D1 leading to cell cycle arrest [56]. The expression level of p16 is significantly reduced in laryngeal carcinoma cells [57]. The gene encoding p16 is localized to 9p21, a region where genetic abbreviations including $\mathrm{LOH}$ are frequently reported [58]. It has been reported that p16 is subjected to the effects of genetic and epigenetic changes such as allelic loss, point mutations, and hypermethylation leading to the high rate of p16 reduction in laryngeal carcinoma [58, 59]. Concurrent loss of heterozygosity and methylation of P16 has also been reported in laryngeal carcinoma [60]. In comparison with other inactivation mechanisms including homozygous deletion, methylation of the promoter region, and point mutation, p16 methylation tends to be the most frequent inactivation pathways found in head and neck cancer [61]. P16 methylation is a frequent event in laryngeal carcinoma and was detected in more than $80 \%$ of the squamous cell cancer of the larynx [62]. Recently, it is demonstrated that methylation of p16 is an early event in squamous cell carcinoma at the laryngopharyngeal region, implying that methylated p16 may have a clinical value in screening patients with early laryngeal carcinoma [63]. Koscielny et al. proposed that methylated $\mathrm{p} 16$ had prognostic value in a followup period of 3 years [64].

2.1.4. DAPK Methylation. DAPK is one of the most frequently methylated genes in laryngeal carcinoma. Park et al. demonstrated the highest methylation frequencies $(87 \%)$ in the laryngeal carcinoma tissues [65]. Similar results have been demonstrated by Calmon et al., and they suggested that they observed an additional correlation of methylated DAPK1 with the lymph node metastasis [66]. Death-associated protein kinase 1 or DAPK is a calcium/calmodulindependent serine/threonine kinase involved in gammainterferon-induced programmed cell death. Methylation of DAPK1 is an early event in the carcinogenesis of head and neck cancers [67]. In lung cancers, DAPK methylation was predominant in older patients in comparison with the young [68]. The methylation status of the promoter region of DAPK gene plays a significant part in controlling DAPK transcription in laryngeal carcinoma. In laryngeal squamous cell cancer with DAPK methylation, DAPK mRNA was totally undetectable [69]; methylated DAPK could be employed to be used as a screening marker in head and neck cancers as it could be detected in the saliva of head and neck cancer patients with potential value in predicting cancer recurrence [70]. Later studies confirmed that methylated DAPK is a good minimal invasive biomarker for head and neck cancer screening as it is detectable in the body fluid of head and neck cancer patients [67].

2.2. DNA Hypomethylation in Laryngeal Carcinoma Cells. As mentioned above, focal methylation of specific tumor suppressor genes is common in cancer cells. The number of methylated tumor suppressor genes is higher in cancer cells in comparison with the analogous normal counterpart. However, if we look at the density of methylated CpG dinucleotides, there is a substantial reduction of 5-methylcytosine content [71]. The global loss of genomic 5-methylcytosine content is recognized as global hypomethylation and is associated with the dysregulation of DNA methyltransferase DNMT1 [72, 73]. DNMT1 is the most highly expressed methyltransferase in somatic cells [74]. It maintains the 5methylcytosine patterns on the newly synthesized DNA after cell division.

An example of hypomethylated genes in laryngeal carcinoma cells is S100A4. S100A4 is an acidic calcium-binding protein and functions as oncogene in a variety of human malignancies. In oral squamous cell carcinoma, S100A4 could suppress expression of the epithelial protein E-cadherin [75]. It could also induce angiogenesis through activation of VEGF expression in oral squamous cells [76]. Results from animal models revealed that cancer cells with high S100A4 levels had higher metastatic rate [77]. The aggressive phenotype is partly contributed by the fact that S100A4 could trigger degradation of extracelluar matrix [78]. In laryngeal carcinoma cells, treatment with demethylating agents could induce expression of S100A4 at both RNA and protein levels [79].

The reasons for DNA hypomethylation remain unclear. It is suggested that the global changes in methylation patterns are associated with the dysregulation of the methylation machinery. With a better understanding of the functional role of different DNA methyltransferases, it is now proposed that the loci-specific focal hypermethylation is linked to the dysregulation of de novo methyltransferase $3 \mathrm{~A}$ and $3 \mathrm{~B}$. On the other hand, the global loss in methylcytosine content 
is resulting from the functional error of DNMT1. Global hypomethylation could lead to activation of oncogenes [80]. It is now recognized that the methylation imbalance resulting from focal methylation and global hypermethylation is the leading cause of inactivation of tumor suppressor genes and activation of oncogenes in cancer cells.

In laryngeal carcinoma, it has been reported that hypomethylation is linked to the polymorphism of the genes regulating DNA methylation including methionine synthase (MTR) and 5, 10-methylenetetrahydrofolate dehydrogenase, 5, 10-methenyltetrahydrofolate cyclohydrolase, 10-formyltetrahydrofolate synthetase (MTHFD1), and methylenetetrahydrofolate reductase (MTHFR). According to Kruszyna et al., patients with 2756AG or GG genotypes on MTR have a higher risk of developing laryngeal carcinoma [81].

\subsection{The Role of Histone Modification in Laryngeal Carcinoma.} Histone is the structural unit of nucleosomes and is important to the packing of DNA. Posttranslational modification of histone (e.g., acetylation, methylation, ubiquitylation, phosphorylation, sumoylation, and ribosylation) could control the activity of the surrounding DNA [82]. Modification on histone protein could affect the protein stability, proteinprotein interaction, protein localization, and DNA binding [83]. Laryngeal carcinoma has a higher expression level of $\mathrm{H} 3$, however, the modification status of the overexpressed histone is not yet evaluated [84]. Takahashi et al. reported that histone $\mathrm{H} 3$; was overexpressed in gastric adenocarcinoma and it was subjected to phosphorylation [85]. Association between histone modification (histone H3 lysine 9 methylation, H3 lysine 4 methylation, H3 lysine 9 acetylation) and the transcriptional regulation of tumor suppressor gene is reported in laryngeal carcinoma [86]. According to the results from Yang et al., histone modification is only a layer of regulation. Histone modification usually works together with other epigenetic mechanisms in controlling gene expression [86].

\subsection{Interplay between Small Noncoding RNA and Laryngeal} Carcinoma. In human genome, the protein-coding portion is only about $2 \%$ [87]. The majority of the non-proteinencoding portion is encoding RNA including PIWI-interacting RNAs, small nucleolar RNAs, transcribed ultraconserved regions, and large intergenic noncoding RNAs [88]. According to the size of the noncoding RNA, they could be classified into short ncRNA, midsize ncRNA, and long ncRNA.

2.5. Dysregulation of Short Noncoding RNA in Laryngeal Carcinoma. PIWI-interacting RNA, transcription initiation RNA, and microRNA are 3 major small noncoding RNA encoded in the human genome. PIWI-interacting RNA (26$31 \mathrm{bp)}$ was discovered in 2006 and functions through interacting with the Argonaute family protein PIWI [89]. PIWIinteracting RNA could control gene expression by modulating locus-specific methylation [90]. However, its role in human cancers remains to be elucidated. Transcription initiation RNA controls gene expression at postinitiation stage of transcription [91]. It is evolutionarily conserved and is associated with the chromatin state [92].

\subsubsection{Candidate Tumor-Suppressing MicroRNA in Laryngeal} Carcinoma. In comparison with the other 2 small noncoding RNAs, the pathway of microRNA biogenesis and function in human cancers is much clearer. MicroRNA is small noncoding RNA that regulates gene expression at posttranscriptional level [93]. Mature microRNAs are short singlestranded molecules, which are about 22 nucleotides long. They function by directly interfering with the target mRNA sequence through complementary interaction. The binding of the microRNA to the $3^{\prime}$ UTR of the target mRNA will hinder the subsequent translation process or promote cleavage of the target mRNA [94]. It is estimated that microRNA is involved in the control of about $60 \%$ protein-coding genes [89].

Head and neck cancer has a distinctive microRNA expression in comparison with their normal counterpart, and the microRNA expression is suggested to play a significant part in the pathogenesis of head and neck cancer [95]. In laryngeal carcinoma, microRNA could be classified into oncogenic microRNA and tumor suppressing microRNA [96]. Tumor-suppressing microRNA should target oncogene under normal circumstance. Lujambio et al. demonstrated that loss/suppression of tumor-suppressing microRNA would promote cancer progression because of the elevation in target oncogene expression [97].

Let-7. Let-7 was discovered in 2000 and is one of the wellstudied tumor suppressing microRNAs [98]. Let-7 is a family of microRNA including let-7a, let-7b, let-7c, let-7d, let-7e, let-7f, let-7 g, let-7i, let-7b*, let-7e*, and microRNA-98 [99]. Expression changes of let-7 family member have been reported in numerous human cancers suggesting that the let- 7 isoform is playing a distinctive function in different cancers. Let-7a could repress RAS and/or c-MYC expression in human cancer cells, and such effects are demonstrated in laryngeal carcinoma recently $[100,101]$. Let-7a could sensitize chemoresistant head and neck cancer cells through modulating stemness genes [102]. Let-7d facilitates the development of epithelial-mesenchymal transition (EMT) traits in oral squamous cell carcinoma [103]. In laryngeal carcinoma, let-7 is a growth inhibitor and could induce apoptosis in laryngeal carcinoma cells [100]. Further, let-7 expression is linked to the sensitivity of cancer cells to radio- and/or chemotherapy [104]. It is noted that not all the let-7 family members are downregulated in cancer cells. Let-7i is preferentially upregulated in laryngeal carcinoma, albeit the functional significance remains unclear [105].

MicroRNA-7. MicroRNA-7 is a proliferation suppressor [106]. There are reports suggesting that microRNA-7 suppression leads to the upregulation of epidermal growth factor receptor expression [107]. Webster et al. confirm the association, and they observed that microRNA 7 is linked to cell cycle progression and cell viability [108]. In vivo studies 
demonstrated that microRNA-7 is suppressed by inflammatory response [109]. In addition, increasing microRNA-7 in cancer cell line through liposomal delivery could inhibit cell division and is suggested to be a potential treatment modality [110]. In head and neck cancer, let-7 insulin-like growth factor 1 receptor (IGF1R) pathway is possibly linked with cancer cell migration and invasion [111]. MicroRNA-7 is a candidate therapeutic agent in laryngeal carcinoma as it could enhance the sensitivity of cancer cells to radiotherapy [112].

MicroRNA-206. Downregulation of microRNA-206 has been reported in breast carcinoma, leiomyoma, lung carcinoma, renal cell carcinoma, and rhabdomyosarcomas [113117]. In laryngeal carcinoma tissues, microRNA-206 expression was significantly reduced in comparison with the normal laryngeal tissues $[118,119]$. Zhang et al. demonstrated that the expression levels of miR-206 are associated with the $\mathrm{T}$ grade, nodal metastasis and clinical stage of patients with laryngeal carcinoma [118]. MicroRNA-206 controls the expression of vascular endothelial growth factor (VEGF) in laryngeal cancer cells. VEGF is a prognostic indicator of laryngeal carcinoma and is correlated with tumor size and lymph node metastasis $[118,120]$. Liu et al. show that miR-206 controls cell migration and invasion through modulating the actin cytoskeleton [121]. MicroRNA-206 could induce apoptosis and inhibit the antiapoptotic pathways such as notch3 [113]. Taken together, miR-206 is suggested to be a candidate tumor-suppressing microRNA in human cancers.

2.5.2. Candidate Oncogenic MicroRNA in Laryngeal Carcinoma. In laryngeal carcinoma, studies on tumor-suppressing microRNA are comparably more than the oncogenic microRNA. One representative example of the oncogenic microRNA identified in laryngeal carcinoma is microRNA21. MicroRNA-21 upregulation was first reported in human glioblastoma in 2005 [122]. Overexpression of microRNA21 has been reported in numerous human malignancies suggesting its importance in the genesis of cancer. MicroRNA-21 overexpression is associated with cancer at advanced stages and might have both diagnostic and prognostic values [123125]. Suppressing microRNA-21 expression in laryngeal carcinoma cell could induce apoptosis and prevent cancer cell invasion by inducing cell cycle arrest [126].

2.5.3. Tumor-Suppressing MicroRNA in Laryngeal Carcinoma-Loss through DNA Methylation. MicroRNA is a kind of epigenetic regulator. MicroRNA itself however is also controled by epigenetic mechanisms including DNA methylation.

Methylated MicroRNA-9. According to microRNA registry miRBase (http://www.mirbase.org/), microRNA 9 has 3 isoforms. They are hsa-mir-9-1 (MI0000466), hsa-mir-9-2 (MI0000467), and hsa-mir-9-3 (MI0000468). In oral and oral pharyngeal carcinoma, microRNA-9 is a candidate tumor suppressor and could control cell proliferation through regulating the PTEN pathway. MicroRNA-9 methylation has recently been reported in oral pharyngeal carcinoma including laryngeal carcinoma [127]. Methylated microRNA-9 could differentiate laryngeal cancer from adjacent histologically normal tissues with high specificity (ranging from 97 to $100 \%)$.

Methylated MicroRNA-137. Downregulation of microRNA137 has been reported in head and neck malignancies [128]. The association between microRNA-137 downregulation and microRNA-137 methylation has been reported in a number of solid cancers including head and neck squamous cell carcinoma [129]. Head and neck squamous cell carcinoma patients with methylated microRNA-137 have poor survival rate [129]. In glioblastoma multiforme cells, expression of microRNA-137 could inhibit cancer cell proliferation [130]. Downregulation of microRNA-137 by DNA methylation is an early event in colorectal cancer [131]. Balaguer suggested that methylated microRNA-137 might have a therapeutic use as reverse expression of microRNA-137 in colorectal cancer cell could inhibit proliferation of colorectal cancer cells [131]. In laryngeal carcinoma, however, the functional roles of microRNA-137 remained to be elucidated.

2.5.4. Midsize Noncoding RNA Associated with Laryngeal Carcinoma. Small nucleolar RNA is involved in the modification and stability of ribosomal RNA [132]. Although its function remains to be clarified, differential expression of small nucleolar RNA is observed in non-small-cell lung cancer and could be employed in cancer screening. In addition, the highly expressed small nucleolar RNA could be detected in the peripheral blood of cancer patients suggesting that it may be useful to be used as a kind of molecular marker for cancer patients [133]. Recently, Mirisola et al. performed a genomewide gene expression profiling of 20 laryngeal carcinoma and showed that 2 small noncoding RNAs, SNORA16A and SNORD14C, were associated with the risk of laryngeal carcinoma development [134].

\section{Conclusion}

Increasing evidence suggests that epigenetic alteration is playing a critical part in the development of head and neck carcinoma. In comparison with squamous cell carcinoma of other head and neck regions, studies on the epigenetic dysregulation on laryngeal carcinoma are relatively fewer and most studies focused on a few of the epigenetic changes in their sample group. With the recent advance in technology such as next-generation sequencing and microarray (e.g., methylation array and microRNA array), we could foresee that epigenetic studies on laryngeal carcinoma will change from candidate gene approach to global high-throughput profiling. This provides an opportunity to explore and dissect the disease from different perspectives. More importantly, suitable epigenetic markers could be identified through the gaining understanding of the disease. 


\section{Conflict of Interests}

The authors state they have no conflict of interests and have received no payment in the preparation of this paper.

\section{Acknowledgments}

This paper was supported by the Seed Funding of Basic Research, The University of Hong Kong, and Li Shu Pui Professorship, The University of Hong Kong.

\section{References}

[1] E. A. Chu and Y. J. Kim, "Laryngeal cancer: diagnosis and preoperative work-up," Otolaryngologic Clinics of North America, vol. 41, no. 4, pp. 673-695, 2008.

[2] D. M. Parkin, F. Bray, J. Ferlay et al., "Global cancer statistics," Ca-A Cancer Journal for Clinicians, vol. 61, no. 2, pp. 69-90, 2011.

[3] R. Siegel, E. Ward, Q. Brawley et al., "Cancer Statistics, 2011: the impact of eliminating socioeconomic and racial disparities on premature cancer deaths," CA Cancer Journal for Clinicians, vol. 61, no. 4, pp. 212-236, 2011.

[4] Hong Kong Cancer Registry, Hong Kong, Hospital Authority, 2011, http://www3.ha.org.hk/cancereg/.

[5] S. T. Mayne, B. Cartmel, V. Kirsh, and W. J. Goodwin, "Alcohol and tobacco use prediagnosis and postdiagnosis, and survival in a cohort of patients with early stage cancers of the oral cavity, pharynx, and larynx," Cancer Epidemiology Biomarkers and Prevention, vol. 18, no. 12, pp. 3368-3374, 2009.

[6] N. D. Freedman, C. C. Abnet, M. F. Leitzmann, A. R. Hollenbeck, and A. Schatzkin, "Prospective investigation of the cigarette smoking-head and neck cancer association by sex," Cancer, vol. 110, no. 7, pp. 1593-1601, 2007.

[7] A. Sapkota, C. C. Hsu, D. Zaridze et al., "Dietary risk factors for squamous cell carcinoma of the upper aerodigestive tract in central and eastern Europe," Cancer Causes and Control, vol. 19, no. 10, pp. 1161-1170, 2008.

[8] J. M. Elwood, J. C. Pearson, D. H. Skippen, and S. M. Jackson, "Alcohol, smoking, social and occupational factors in the aetiology of cancer of the oral cavity, pharynx and larynx," International Journal of Cancer, vol. 34, no. 5, pp. 603-612, 1984.

[9] J. A. Koufman, T. A. Radomski, G. M. Joharji, G. B. Russell, and D. C. Pillsbury, "Laryngeal biomechanics of the singing voice,” Otolaryngology, vol. 115, no. 6, pp. 527-537, 1996.

[10] M. C. Torrente, J. P. Rodrigo, M. Haigentz Jr. et al., "Human papillomavirus infections in laryngeal cancer," Head and Neck, vol. 33, no. 4, pp. 581-586, 2011.

[11] A. R. Kreimer, G. M. Clifford, P. Boyle, and S. Franceschi, "Human papillomavirus types in head and neck squamous cell carcinomas worldwide: a systemic review," Cancer Epidemiology Biomarkers and Prevention, vol. 14, no. 2, pp. 467475, 2005.

[12] K. B. Ribeiro, J. E. Levi, M. Pawlita et al., "Low human papillomavirus prevalence in head and neck cancer: results from two large case-control studies in high-incidence regions," International Journal of Epidemiology, vol. 40, no. 2, pp. 489502, 2011.

[13] X. Z. Huang, J. B. Wang, and W. J. Kong, Practice of Otorhinolaryngology-Head and Neck Surgery, 2008, pp. 488-493.
[14] A. Ferlito, K. O. Devaney, and J. Woolgar, "Squamous epithelial changes of the larynx: diagnosis and therapy," Head and Neck. In press.

[15] E. S. Diala, M. S. Cheah, D. Rowitch, and R. M. Hoffman, "Extent of DNA methylation in human tumor cells," Journal of the National Cancer Institute, vol. 71, no. 4, pp. 755-764, 1983.

[16] C. T. Wu and J. R. Morris, "Genes, genetics, and epigenetics: a correspondence," Science, vol. 293, no. 5532, pp. 1103-1105, 2001.

[17] S. L. Berger, T. Kouzarides, R. Shiekhattar, and A. Shilatifard, "An operational definition of epigenetics," Genes and Development, vol. 23, no. 7, pp. 781-783, 2009.

[18] S. Guil and M. Esteller, "DNA methylomes, histone codes and miRNAs: tying it all together," International Journal of Biochemistry and Cell Biology, vol. 41, no. 1, pp. 87-95, 2009.

[19] J. S. Griffith and H. R. Mahler, "DNA ticketing theory of memory," Nature, vol. 223, no. 5206, pp. 580-582, 1969.

[20] R. D. Hotchkiss, "The quantitative separation of purines, pyrimidines, and nucleosides by paper chromatography," The Journal of Biological Chemistry, vol. 175, no. 1, pp. 315-332, 1948.

[21] G. C. Prendergast and E. B. Ziff, "Methylation-sensitive sequence-specific DNA binding by the c-Myc basic region," Science, vol. 251, no. 4990, pp. 186-189, 1991.

[22] M. Hatziapostolou and D. Iliopoulos, "Epigenetic aberrations during oncogenesis," Cellular and Molecular Life Sciences, vol. 68, no. 10, pp. 1681-1702, 2011.

[23] O. Bogdanović and G. J. Veenstra, "DNA methylation and methyl-CpG binding proteins: developmental requirements and function," Chromosoma, vol. 118, no. 5, pp. 549-565, 2009.

[24] S. Pradhan, J. K. Kim, and M. Samaranayake, "Epigenetic mechanisms in mammals," Cellular and Molecular Life Sciences, vol. 66, no. 4, pp. 596-612, 2009.

[25] K. M. Kim and D. Shibata, "Methylation reveals a niche: stem cell succession in human colon crypts," Oncogene, vol. 21, no. 35, pp. 5441-5449, 2002.

[26] A. L. Reed, J. Califano, P. Cairns et al., "High frequency of p16 (CDKN2/MTS-1/INK4A) inactivation in head and neck squamous cell carcinoma," Cancer Research, vol. 56, no. 16, pp. 3630-3633, 1996.

[27] L. Ai, Q. N. Vo, C. Zuo et al., "Ataxia-telangiectasia-mutated (ATM) gene in head and neck squamous cell carcinoma: promoter hypermethylation with clinical correlation in 100 cases," Cancer Epidemiology Biomarkers and Prevention, vol. 13, no. 1, pp. 150-156, 2004.

[28] K. Ogi, M. Toyota, M. Ohe-Toyota et al., "Aberrant methylation of multiple genes and clinicopathological features in oral squamous cell carcinoma," Clinical Cancer Research, vol. 8, no. 10, pp. 3164-3171, 2002.

[29] E. M. Youssef, D. Lotan, J. P. Issa et al., "Hypermethylation of the retinoic acid receptor- $\beta 2$ gene in head and neck carcinogenesis," Clinical Cancer Research, vol. 10, no. 5, pp. 17331742, 2004.

[30] R. J. Shaw, T. Liloglou, S. N. Rogers et al., "Promoter methylation of P16, RAR $\beta$, E-cadherin, cyclin A1 and cytoglobin in oral cancer: quantitative evaluation using pyrosequencing," British Journal of Cancer, vol. 94, no. 4, pp. 561-568, 2006.

[31] S. M. Dong, D. I. Sun, N. E. Benoit, I. Kuzmin, M. I. Lerman, and D. Sidransky, "Epigenetic inactivation of RASSF1A in head and neck cancer," Clinical Cancer Research, vol. 9, no. 10, part 1, pp. 3635-3640, 2003. 
[32] M. R. H. Estécio, E. M. Youssef, P. Rahal et al., "LHX6 is a sensitive methylation marker in head and neck carcinomas," Oncogene, vol. 25, no. 36, pp. 5018-5026, 2006.

[33] H. W. Chang, V. Chow, K. Y. Lam, W. I. Wei, and A. P. WingYuen, "Loss of E-cadherin expression resulting from promoter hypermethylation in oral tongue carcinoma and its prognostic significance," Cancer, vol. 94, no. 2, pp. 386-392, 2002.

[34] M. Viswanathan, N. Tsuchida, and G. Shanmugam, "Promoter hypermethylation profile of tumor-associated genes p16, p15, hMLH1, MGMT and E-cadherin in oral squamous cell carcinoma," International Journal of Cancer, vol. 105, no. 1, pp. 41-46, 2003.

[35] D. T. Cody II, Y. Huang, C. J. Darby, G. K. Johnson, and F. E. Domann, "Differential DNA methylation of the p16 INK4A/ CDKN2A promoter in human oral cancer cells and normal human oral keratinocytes," Oral Oncology, vol. 35, no. 5, pp. 516-522, 1999.

[36] P. M. Thompson, T. Gotoh, M. Kok, P. S. White, and G. M. Brodeur, "CHD5, a new member of the chromodomain gene family, is preferentially expressed in the nervous system," Oncogene, vol. 22, no. 7, pp. 1002-1011, 2003.

[37] H. Koyama, T. Zhuang, and J. E. Light, "Mechanisms of CHD5 inactivation in neuroblastomas," Clinical Cancer Research, vol. 18, no. 6, pp. 1588-1597, 2012.

[38] W. Owens, J. K. Field, P. J. Howard, and P. M. Stell, "Multiple cytogenetic aberrations in squamous cell carcinomas of the head and neck," European Journal of Cancer B, vol. 28, no. 1, pp. 17-21, 1992.

[39] A. K. El-Naggar, S. Lai, G. L. Clayman et al., "P73 gene alterations and expression in primary oral and laryngeal squamous carcinomas," Carcinogenesis, vol. 22, no. 5, pp. 729 735, 2001.

[40] J. Wang, H. Chen, and S. Fu, "The involvement of CHD5 hypermethylation in laryngeal squamous cell carcinoma," Oral Oncology, vol. 47, no. 7, pp. 601-608, 2011.

[41] A. Bagchi, C. Papazoglu, Y. Wu et al., "CHD5 Is a tumor suppressor at human 1p36," Cell, vol. 128, no. 3, pp. 459-475, 2006.

[42] M. Takeichi, "Cadherin cell adhesion receptors as a morphogenetic regulator," Science, vol. 251, no. 5000, pp. 1451-1455, 1991.

[43] M. M. Nijkamp, P. N. Span, I. J. Hoogsteen, A. J. Van Der Kogel, J. H.A.M. Kaanders, and J. Bussink, "Expression of Ecadherin and vimentin correlates with metastasis formation in head and neck squamous cell carcinoma patients," Radiotherapy and Oncology, vol. 99, no. 3, pp. 344-348, 2011.

[44] D. Wang, L. Su, D. Huang, H. Zhang, D. M. Shin, and Z. G. Chen, "Downregulation of E-Cadherin enhances proliferation of head and neck cancer through transcriptional regulation of EGFR," Molecular Cancer, vol. 22, no. 10, p. 116, 2011.

[45] J. P. Rodrigo, F. Domínguez, C. Alvarez, C. Manrique, A. Herrero, and C. Suárez, "Expression of E-cadherin in squamous cell carcinomas of the supraglottic larynx with correlations to clinicopathological features," European Journal of Cancer, vol. 38, no. 8, pp. 1059-1064, 2002.

[46] O. Reichel, D. Mayr, F. Durst, and A. Berghaus, "E-cadherin but not $\beta$-catenin expression is decreased in laryngeal biopsies from patients with laryngopharyngeal reflux," European Archives of Oto-Rhino-Laryngology, vol. 265, no. 8, pp. 937$942,2008$.
[47] H. Galera-Ruiz, M. J. Ríos-Moreno, and R. GonzálezCámpora, "The cadherin-catenin complex in laryngeal squamous cell carcinoma," European Archives of Otorhinolaryngology, vol. 269, no. 4, pp. 1183-1188, 2012.

[48] E. Mittari, A. Charalabopoulos, A. Batistatou, and K. Charalabopoulos, "The role of E-cadherin/catenin complex in laryngeal cancer," Experimental Oncology, vol. 27, no. 4, pp. 257-261, 2005.

[49] J. P. Rodrigo, F. Dominguez, V. Suárez, M. Canel, P. Secades, and M. D. Chiara, "Focal adhesion kinase and E-cadherin as markers for nodal metastasis in laryngeal cancer," Archives of Otolaryngology, vol. 133, no. 2, pp. 145-150, 2007.

[50] M. Paksoy, U. Hardal, and C. Caglar, "Expression of cathepsin D and E-cadherin in primary laryngeal cancers correlation with neck lymph node involvement," Journal of Cancer Research and Clinical Oncology, vol. 137, no. 9, pp. 13711377, 2011.

[51] J. J. Li, G. H. Zhang, X. M. Yang et al., "Reduced E-cadherin expression is associated with lymph node metastases in laryngeal squamous cell carcinoma," Auris Nasus Larynx, vol. 39, no. 2, pp. 186-192, 2012.

[52] J. G. Eriksen, F. M. Buffa, J. Alsner, T. Steiniche, S. M. Bentzen, and J. Overgaard, "Molecular profiles as predictive marker for the effect of overall treatment time of radiotherapy in supraglottic larynx squamous cell carcinomas," Radiotherapy and Oncology, vol. 72, no. 3, pp. 275-282, 2004.

[53] P. Azarschab, A. Stembalska, M. B. Loncar, M. Pfister, M. M. Sasiadek, and N. Blin, "Epigenetic control of E-cadherin $(\mathrm{CDH} 1)$ by $\mathrm{CpG}$ methylation in metastasising laryngeal cancer," Oncology Reports, vol. 10, no. 2, pp. 501-503, 2003.

[54] R. P. Dikshit, A. Gillio-Tos, P. Brennan et al., "Hypermethylation, risk factors, clinical characteristics, and survival in 235 patients with laryngeal and hypopharyngeal cancers," Cancer, vol. 110, no. 8, pp. 1745-1751, 2007.

[55] C. J. Marsit, M. R. Posner, M. D. McClean, and K. T. Kelsey, "Hypermethylation of E-cadherin is an independent predictor of improved survival in head and neck squamous cell carcinoma," Cancer, vol. 113, no. 7, pp. 1566-1571, 2008.

[56] C. J. Sherr, "Cancer cell cycles," Science, vol. 274, no. 5293, pp. 1672-1674, 1996.

[57] A. K. El-Naggar, S. Lai, G. L. Clayman et al., "Expression of $\mathrm{p} 16, \mathrm{Rb}$, and cyclin D1 gene products in oral and laryngeal squamous carcinoma: biological and clinical implications," Human Pathology, vol. 30, no. 9, pp. 1013-1018, 1999.

[58] P. Jares, A. Nadal, P. L. Fernández et al., "Disregulation of p16(MTS1/CDK4I) protein and mRNA expression is associated with gene alterations in squamous-cell carcinoma of the larynx," International Journal of Cancer, vol. 81, no. 5, pp. 705-711, 1999.

[59] S. Bai, H. Gao, H. Liang, S. Fei, X. Zhang, and K. Sun, “The study of p16 gene mutation and methylation in laryngeal squamous cell carcinoma," Chinese Journal of Otorhinolaryngology, Head and Neck Surgery, vol. 35, no. 4, pp. 292-294, 2000.

[60] M. M. Sasiadek, A. Stembalska-Kozlowska, R. Smigiel, D. Ramsey, T. Kayademir, and N. Blin, "Impairment of MLHI and CDKN2A in oncogenesis of laryngeal cancer," British Journal of Cancer, vol. 90, no. 8, pp. 1594-1599, 2004.

[61] U. Riese, R. Dahse, W. Fiedler et al., "Tumor suppressor gene p16 (CDKN2A) mutation status and promoter inactivation in head and neck cancer," International Journal of Molecular Medicine, vol. 4, no. 1, pp. 61-65, 1999. 
[62] R. Smigiel, M. Sasiadek, T. Krecicki, D. Ramsey, J. Jagielski, and N. Blin, "Inactivation of the cyclin-dependent kinase inhibitor 2A (CDKN2A) gene in squamous cell carcinoma of the larynx," Molecular Carcinogenesis, vol. 39, no. 3, pp. 147$154,2004$.

[63] S. Temam, J. Bénard, C. Dugas et al., "Molecular detection of early-stage laryngopharyngeal squamous cell carcinomas," Clinical Cancer Research, vol. 11, no. 7, pp. 2547-2551, 2005.

[64] S. Koscielny, R. Dahse, G. Ernst, and F. Von Eggeling, "The prognostic relevance of p16 inactivation in head and neck cancer," ORL, vol. 69, no. 1, pp. 30-36, 2006.

[65] S. Y. Park, B. H. Kim, H. K. Jeong et al., "Methylation profiles of CpG island loci in major types of human cancers," Journal of Korean Medical Science, vol. 22, no. 2, pp. 311-317, 2007.

[66] M. F. Calmon, J. Colombo, F. Carvalho et al., "Methylation profile of genes CDKN2A (p14 and p16), DAPK1, CDH1, and ADAM23 in head and neck cancer," Cancer Genetics and Cytogenetics, vol. 173, no. 1, pp. 31-37, 2007.

[67] Y. Liu, Z. T. Zhou, Q. B. He et al., "DAPK promoter hypermethylation in tissues and body fluids of oral precancer patients," Medical Oncology. In press.

[68] J. C. Soria, M. Rodriguez, D. D. Liu, J. Jack Lee, W. Ki Hong, and L. Mao, "Aberrant promoter methylation of multiple genes in bronchial brush samples from former cigarette smokers," Cancer Research, vol. 62, no. 2, pp. 351-355, 2002.

[69] S. Zhang and W. J. Kong, "Hypermethylation of the deathassociated protein kinase promoter in laryngeal squamous cell cancer," Chinese Journal of Oncology, vol. 26, no. 8, pp. 469-471, 2004.

[70] C. A. Righini, F. de Fraipont, J. F. Timsit et al., "Tumorspecific methylation in saliva: a promising biomarker for early detection of head and neck cancer recurrence," Clinical Cancer Research, vol. 13, no. 4, pp. 1179-1185, 2007.

[71] O. Bruserud, C. Stapnes, E. Ersvaer, B. T. Gjertsen, and A. Ryningen, "Histone deacetylase inhibitors in cancer treatment: a review of the clinical toxicity and the modulation of gene expression in cancer cells," Current Pharmaceutical Biotechnology, vol. 8, no. 6, pp. 388-400, 2007.

[72] F. Gaudet, J. G. Hodgson, A. Eden et al., "Induction of tumors in mice by genomic hypomethylation," Science, vol. 300, no. 5618, pp. 489-492, 2003.

[73] B. K. Dunn, "Hypomethylation: one side of a larger picture," Annals of the New York Academy of Sciences, vol. 983, pp. 2842, 2003.

[74] P. A. Jones and G. Liang, "Rethinking how DNA methylation patterns are maintained," Nature Reviews Genetics, vol. 10, no. 11, pp. 805-811, 2009.

[75] M. M. Kita, Y. Endo, Y. Yonemura et al., "S100A4 regulates Ecadherin expression in oral squamous cell carcinoma," Cancer Letters, vol. 230, no. 2, pp. 211-218, 2005.

[76] R. Sedivy, J. B. Mannagetta, C. Haverkampf, W. Battistutti, and S. Hönigschnabl, "Expression of vascular endothelial growth factor-C correlates with the lymphatic microvessel density and the nodal status in oral squamous cell cancer," Journal of Oral Pathology and Medicine, vol. 32, no. 8, pp. 455-460, 2003.

[77] D. Levett, P. A. Flecknell, P. S. Rudland et al., "Transfection of S100A4 produces metastatic variants of an orthotopic model of bladder cancer," The American Journal of Pathology, vol. 160, no. 2, pp. 693-700, 2002.

[78] M. Saleem, M. H. Kweon, J. J. Johnson et al., "S100A4 accelerates tumorigenesis and invasion of human prostate cancer through the transcriptional regulation of matrix metalloproteinase 9," Proceedings of the National Academy of Sciences of the United States of America, vol. 103, no. 40, pp. 1482514830, 2006.

[79] J. Liu, Y. Guo, S. Fu, M. Yang, K. L. Sun, and W. N. Fu, "Hypomethylation-induced expression of S100A4 increases the invasiveness of laryngeal squamous cell carcinoma," Oncology Reports, vol. 23, no. 4, pp. 1101-1107, 2010.

[80] W. Sun, Y. Liu, C. A. Glazer et al., "TKTL1 is activated by promoter hypomethylation and contributes to head and neck squamous cell carcinoma carcinogenesis through increased aerobic glycolysis and HIF1 $\alpha$ stabilization," Clinical Cancer Research, vol. 16, no. 3, pp. 857-866, 2010.

[81] Ł. Kruszyna, M. Lianeri, M. Rydzanicz, M. Gajȩcka, K. Szyfter, and P. P. Jagodziński, "Polymorphic variants of folate metabolism genes and the risk of laryngeal cancer," Molecular Biology Reports, vol. 37, no. 1, pp. 241-247, 2010.

[82] T. Jenuwein and C. D. Allis, "Translating the histone code," Science, vol. 293, no. 5532, pp. 1074-1080, 2001.

[83] S. Minucci and P. G. Pelicci, "Histone deacetylase inhibitors and the promise of epigenetic (and more) treatments for cancer," Nature Reviews Cancer, vol. 6, no. 1, pp. 38-51, 2006.

[84] M. Kapral, B. Strzalka-Mrozik, M. Kowalczyk et al., "Transcriptional activities of histone H3, cyclin D1 and claudin 7 encoding genes in laryngeal cancer," European Archives of Oto-Rhino-Laryngology, vol. 268, no. 5, pp. 709-714, 2011.

[85] H. Takahashi, Y. Murai, K. Tsuneyama et al., "Overexpression of phosphorylated histone $\mathrm{H} 3$ is an indicator of poor prognosis in gastric adenocarcinoma patients," Applied Immunohistochemistry and Molecular Morphology, vol. 14, no. 3, pp. 296-302, 2006.

[86] J. Yang, W. Y. Ji, Y. R. Qu, L. X. He, X. D. Zhao, and M. Z. Jin, "DNA methylation and histone modification relate to RASSF1A gene deletion in laryngeal carcinoma tissues," Chinese Journal of Otorhinolaryngology Head and Neck Surgery, vol. 46, no. 4, pp. 308-312, 2011.

[87] R. P. Alexander, G. Fang, J. Rozowsky, M. Snyder, and M. B. Gerstein, "Annotating non-coding regions of the genome," Nature Reviews Genetics, vol. 11, no. 8, pp. 559-571, 2010.

[88] M. Esteller, "Non-coding RNAs in human disease," Nature Reviews Genetics, vol. 12, no. 12, pp. 861-874, 2011.

[89] J. Brennecke, A. A. Aravin, A. Stark et al., "Discrete small RNA-generating loci as master regulators of transposon activity in Drosophila," Cell, vol. 128, no. 6, pp. 1089-1103, 2007.

[90] T. Watanabe, S. Tomizawa, K. Mitsuya et al., "Role for piRNAs and noncoding RNA in de novo DNA methylation of the imprinted mouse Rasgrf1 locus," Science, vol. 332, no. 6031, pp. 848-852, 2011.

[91] J. Mellor, P. Dudek, and D. Clynes, "A glimpse into the epigenetic landscape of gene regulation," Current Opinion in Genetics and Development, vol. 18, no. 2, pp. 116-122, 2008.

[92] R. J. Taft, E. A. Glazov, N. Cloonan et al., "Tiny RNAs associated with transcription start sites in animals," Nature Genetics, vol. 41, no. 5, pp. 572-578, 2009.

[93] L. He and G. J. Hannon, "MicroRNAs: small RNAs with a big role in gene regulation," Nature Reviews Genetics, vol. 5, no. 7, pp. 522-531, 2004.

[94] D. P. Bartel, "MicroRNAs: genomics, biogenesis, mechanism, and function," Cell, vol. 116, no. 2, pp. 281-297, 2004.

[95] M. Shiiba, K. Uzawa, and H. Tanzawa, "MicroRNAs in head and neck squamous cell carcinoma (HNSCC) and oral squamous cell carcinoma (OSCC)," Cancers, vol. 2, no. 2, pp. 653$669,2010$.

[96] C. Z. Chen, "MicroRNAs as oncogenes and tumor suppressors," The New England Journal of Medicine, vol. 353, no. 17, pp. 1768-1771, 2005. 
[97] A. Lujambio, G. A. Calin, A. Villanueva et al., "A microRNA DNA methylation signature for human cancer metastasis," Proceedings of the National Academy of Sciences of the United States of America, vol. 105, no. 36, pp. 13556-13561, 2008.

[98] B. Boyerinas, S. M. Park, A. Hau, A. E. Murmann, and M. E. Peter, "The role of let-7 in cell differentiation and cancer," Endocrine-Related Cancer, vol. 17, no. 1, pp. 19-36, 2010.

[99] B. J. Reinhart, F. J. Slack, M. Basson et al., "The 21-nucleotide let-7 RNA regulates develop- mental timing in Caenorhabditis elegans," Nature, vol. 403, no. 6772, pp. 901-906, 2000.

[100] X. B. Long, G. B. Sun, S. Hu et al., "Let-7a microrna functions as a potential tumor suppressor in human laryngeal cancer," Oncology Reports, vol. 22, no. 5, pp. 1189-1195, 2009.

[101] V. B. Sampson, N. H. Rong, J. Han et al., "MicroRNA let7a down-regulates MYC and reverts MYC-induced growth in Burkitt lymphoma cells," Cancer Research, vol. 67, no. 20, pp. 9762-9770, 2007.

[102] C. C. Yu, Y. W. Chen, G. Y. Chiou et al., "MicroRNA let-7a represses chemoresistance and tumourigenicity in head and neck cancer via stem-like properties ablation," Oral Oncology, vol. 47, no. 3, pp. 202-210, 2011.

[103] C. J. Chang, C. C. Hsu, C. H. Chang et al., "Let-7d functions as novel regulator of epithelial-mesenchymal transition and chemoresistant property in oral cancer," Oncology Reports, vol. 26, no. 4, pp. 1003-1010, 2011.

[104] R. Hummel, D. J. Hussey, and J. Haier, "MicroRNAs: predictors and modifiers of chemo- and radiotherapy in different tumour types," European Journal of Cancer, vol. 46, no. 2, pp. 298-311, 2010.

[105] S. S. Chang, W. J. Wei, I. Smith et al., "MicroRNA alterations in head and neck squamous cell carcinoma," International Journal of Cancer, vol. 123, no. 12, pp. 2791-2797, 2008.

[106] Y. X. Fang, J. L. Xue, and L. Tian, "miR-7 inhibits tumor growth and metastasis by targeting the PI3K/AKT pathway in hepatocellular carcinoma," Hepatology. In press.

[107] B. Kefas, J. Godlewski, L. Comeau et al., "microRNA-7 inhibits the epidermal growth factor receptor and the akt pathway and is down-regulated in glioblastoma," Cancer Research, vol. 68, no. 10, pp. 3566-3572, 2008.

[108] R. J. Webster, K. M. Giles, K. J. Price, P. M. Zhang, J. S. Mattick, and P. J. Leedman, "Regulation of epidermal growth factor receptor signaling in human cancer cells by MicroRNA-7," Journal of Biological Chemistry, vol. 284, no. 9, pp. 5731-5741, 2009.

[109] D. Kong, Y. S. Piao, S. Yamashita et al., "Inflammation-induced repression of tumor suppressor miR-7 in gastric tumor cells," Oncogene. In press.

[110] K. Rai, N. Takigawa, S. Ito et al., "Liposomal delivery of MicroRNA-7-expressing plasmid overcomes epidermal growth factor receptor tyrosine kinase inhibitor-resistance in lung cancer cells," Molecular Cancer Therapeutics, vol. 10, no. 9, pp. 1720-1727, 2011.

[111] L. Jiang, X. Liu, Z. Chen et al., "MicroRNA-7 targets IGF1R (insulin-like growth factor 1 receptor) in tongue squamous cell carcinoma cells," Biochemical Journal, vol. 432, no. 1, pp. 199-205, 2010.

[112] K. M. Lee, E. J. Choi, and I. A. Kim, "MicroRNA-7 increases radiosensitivity of human cancer cells with activated EGFRassociated signaling," Radiotherapy and Oncology, vol. 101, no. 1, pp. 171-176, 2011.
[113] G. Song, Y. Zhang, and L. Wang, "MicroRNA-206 targets notch3, activates apoptosis, and inhibits tumor cell migration and focus formation," Journal of Biological Chemistry, vol. 284, no. 46, pp. 31921-31927, 2009.

[114] X. Wang, C. Ling, Y. Bai, and J. Zhao, "MicroRNA-206 is associated with invasion and metastasis of lung cancer," Anatomical Record, vol. 294, no. 1, pp. 88-92, 2011.

[115] Q. Pan, X. Luo, and N. Chegini, "Differential expression of microRNAs in myometrium and leiomyomas and regulation by ovarian steroids: in focus," Journal of Cellular and Molecular Medicine, vol. 12, no. 1, pp. 227-240, 2008.

[116] E. Missiaglia, C. J. Shepherd, S. Patel et al., "MicroRNA-206 expression levels correlate with clinical behaviour of rhabdomyosarcomas," British Journal of Cancer, vol. 102, no. 12, pp. 1769-1777, 2010.

[117] H. Hidaka, N. Seki, H. Yoshino et al., "Tumor suppressive microRNA-1285 regulates novel molecular targets: aberrant expression and functional significance in renal cell carcinoma," Oncotarget, vol. 3, no. 1, pp. 44-57, 2012.

[118] T. Zhang, M. Liu, C. Wang, C. Lin, Y. Sun, and D. Jin, "Down-regulation of MiR-206 promotes proliferation and invasion of laryngeal cancer by regulating VEGF expression," Anticancer Research, vol. 31, no. 11, pp. 3859-3863, 2011.

[119] P. Wang, T. Fu, X. Wang, and W. Zhu, "Primary, study of miRNA expression patterns in laryngeal carcinoma by microarray," Journal of Clinical Otorhinolaryngology, Head, and Neck Surgery, vol. 24, no. 12, pp. 535-538, 2010.

[120] Y. Sullu, S. Gun, S. Atmaca, F. Karagoz, and B. Kandemir, "Poor prognostic clinicopathologic features correlate with VEGF expression but not with PTEN expression in squamous cell carcinoma of the larynx," Diagnostic Pathology, vol. 14, no. 5, p. 35, 2010.

[121] H. Liu, Y. D. Cao, W. X. Ye, and Y. Y. Sun, "Effect of microRNA-206 on cytoskeleton remodelling by downregulating Cdc42 in MDA-MB-231 cells," Tumori, vol. 96, no. 5, pp. 751-755, 2010.

[122] J. A. Chan, A. M. Krichevsky, and K. S. Kosik, "MicroRNA21 is an antiapoptotic factor in human glioblastoma cells," Cancer Research, vol. 65, no. 14, pp. 6029-6033, 2005.

[123] L. X. Yan, X. F. Huang, Q. Shao et al., "MicroRNA miR-21 overexpression in human breast cancer is associated with advanced clinical stage, lymph node metastasis and patient poor prognosis," RNA, vol. 14, no. 11, pp. 2348-2360, 2008.

[124] C. H. Lawrie, S. Soneji, T. Marafioti et al., "MicroRNA expression distinguishes between germinal center B cell-like and activated B cell-like subtypes of diffuse large B cell lymphoma," International Journal of Cancer, vol. 121, no. 5, pp. 1156-1161, 2007.

[125] S. H. Chan, C. W. Wu, A. F. Y. Li, C. W. Chi, and W. C. Lin, "miR-21 microRNA expression in human gastric carcinomas and its clinical association," Anticancer Research A, vol. 28, no. 2, pp. 907-911, 2008.

[126] J. Ren, D. Zhu, M. Liu, Y. Sun, and L. Tian, “Downregulation of miR-21 modulates Ras expression to promote apoptosis and suppress invasion of Laryngeal squamous cell carcinoma," European Journal of Cancer, vol. 46, no. 18, pp. 34093416, 2010.

[127] J. Minor, X. Wang, F. Zhang et al., "Methylation of microRNA-9 is a specific and sensitive biomarker for oral and oropharyngeal squamous cell carcinomas," Oral Oncology, vol. 48 , no. 1, pp. 73-78, 2012. 
[128] T. S. Wong, X. B. Liu, B. Y. H. Wong, R. W. M. Ng, A. P. W. Yuen, and W. I. Wei, "Mature miR-184 as potential oncogenic microRNA of squamous cell carcinoma of tongue," Clinical Cancer Research, vol. 14, no. 9, pp. 2588-2592, 2008.

[129] S. M. Langevin, R. A. Stone, C. H. Bunker et al., "MicroRNA137 promoter methylation is associated with poorer overall survival in patients with squamous cell carcinoma of the head and neck," Cancer, vol. 117, no. 7, pp. 1454-1462, 2011.

[130] J. Silber, D. A. Lim, C. Petritsch et al., "miR-124 and miR-137 inhibit proliferation of glioblastoma multiforme cells and induce differentiation of brain tumor stem cells," BMC Medicine, vol. 6, no. 14, pp. 1-17, 2008.

[131] F. Balaguer, A. Link, J. J. Lozano et al., "Epigenetic silencing of miR-137 is an early event in colorectal carcinogenesis," Cancer Research, vol. 70, no. 16, pp. 6609-6618, 2010.

[132] T. H. King, B. Liu, R. R. McCully, and M. J. Fournier, "Ribosome structure and activity are altered in cells lacking snoRNPs that form pseudouridines in the peptidyl transferase center," Molecular Cell, vol. 11, no. 2, pp. 425-435, 2003.

[133] J. Liao, L. Yu, Y. Mei et al., "Small nucleolar RNA signatures as biomarkers for non-small-cell lung cancer," Molecular Cancer, vol. 7, no. 9, p. 198, 2010.

[134] V. Mirisola, R. Mora, A. I. Esposito et al., "A prognostic multigene classifier for squamous cell carcinomas of the larynx," Cancer Letters, vol. 307, no. 1, pp. 37-46, 2011. 


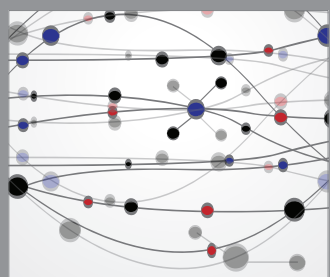

The Scientific World Journal
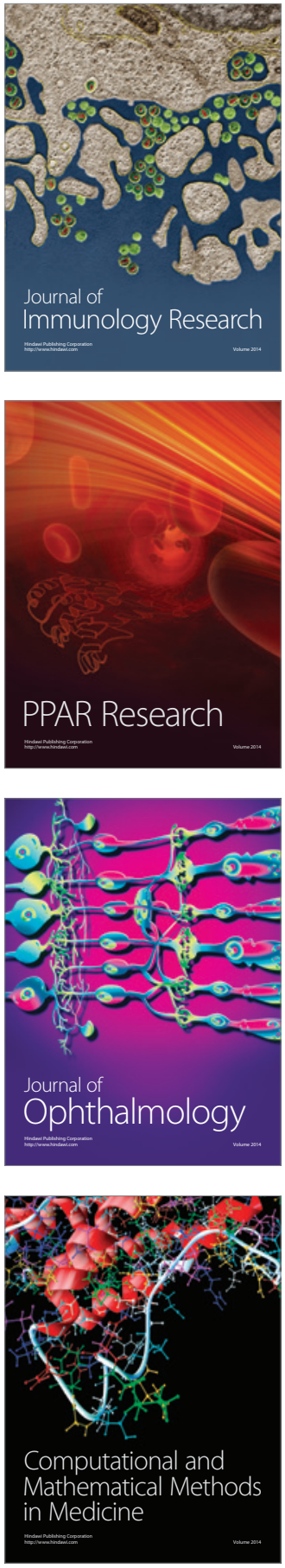

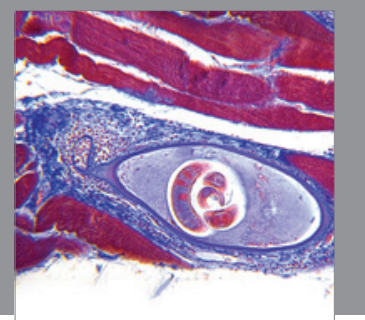

Gastroenterology

Research and Practice
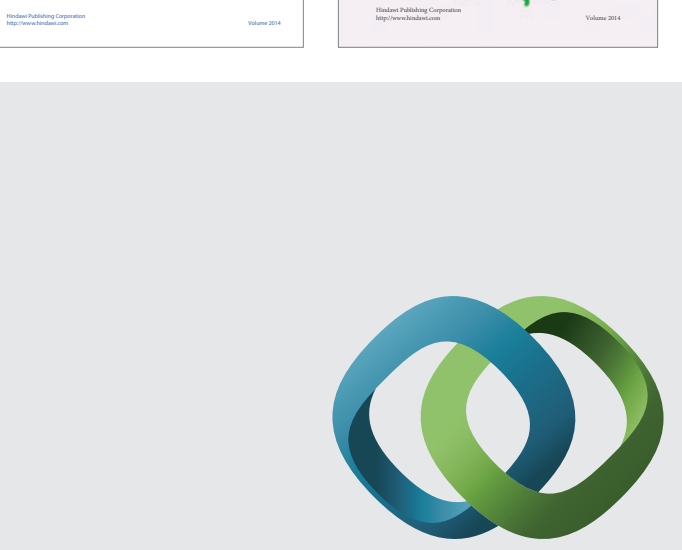

\section{Hindawi}

Submit your manuscripts at

http://www.hindawi.com
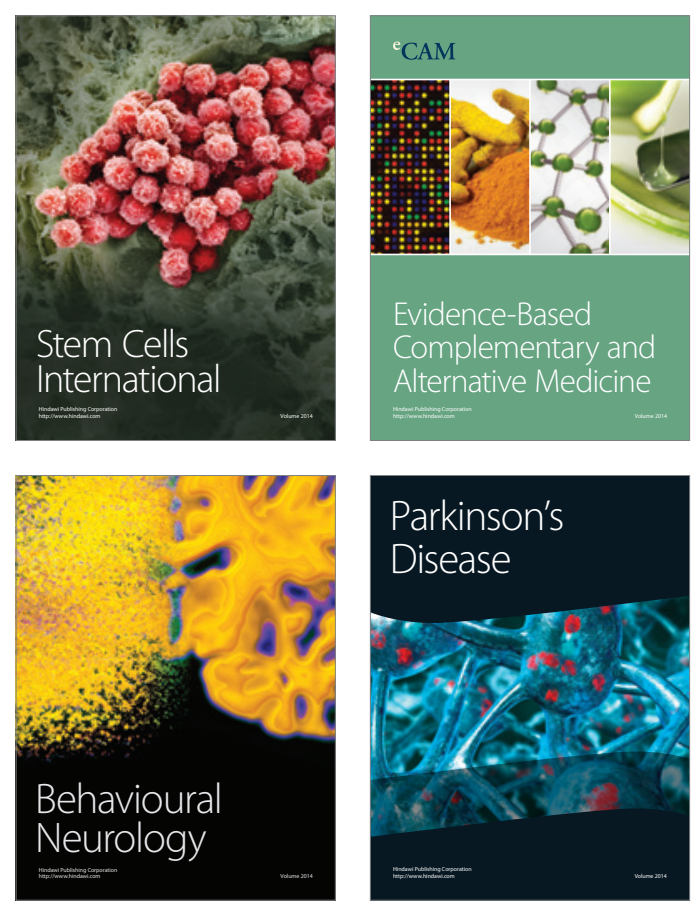

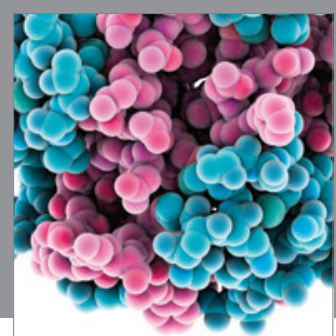

Journal of
Diabetes Research

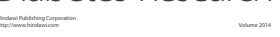

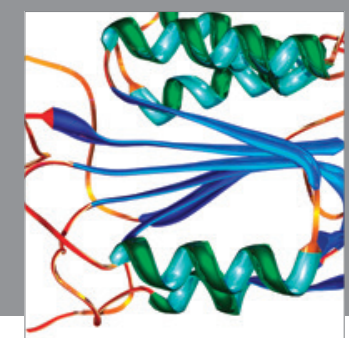

Disease Markers
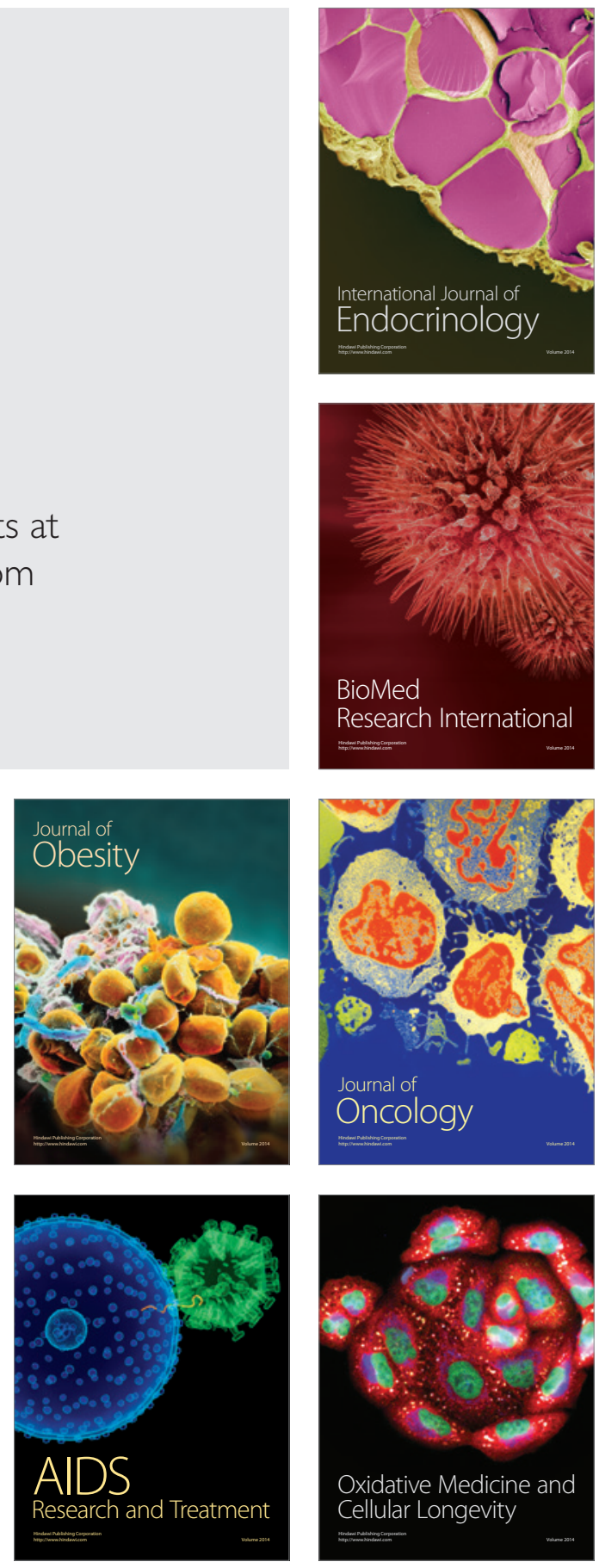